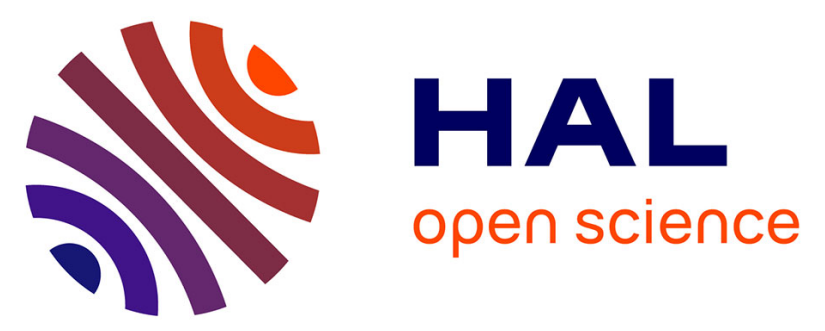

\title{
MRI findings and clinical outcome in 45 divers with spinal cord decompression sickness.
}

Emmanuel Gempp, Jean-Eric Blatteau, Eric Stephant, Jean-Michel Pontier, Pascal Constantin, Christophe Pény

\section{- To cite this version:}

Emmanuel Gempp, Jean-Eric Blatteau, Eric Stephant, Jean-Michel Pontier, Pascal Constantin, et al.. MRI findings and clinical outcome in 45 divers with spinal cord decompression sickness.. Aviation, Space, and Environmental Medicine, 2008, 79 (12), pp.1112-6. ssa-00355981

\section{HAL Id: ssa-00355981}

\section{https://hal-ssa.archives-ouvertes.fr/ssa-00355981}

Submitted on 26 Jan 2009

HAL is a multi-disciplinary open access archive for the deposit and dissemination of scientific research documents, whether they are published or not. The documents may come from teaching and research institutions in France or abroad, or from public or private research centers.
L'archive ouverte pluridisciplinaire HAL, est destinée au dépôt et à la diffusion de documents scientifiques de niveau recherche, publiés ou non, émanant des établissements d'enseignement et de recherche français ou étrangers, des laboratoires publics ou privés. 
MRI FINDINGS AND CLINICAL OUTCOME IN 45 DIVERS WITH SPINAL CORD DECOMPRESSION SICKNESS

Emmanuel Gempp $\mathrm{MD}^{1}$, Jean-Eric Blatteau $\mathrm{MD} \mathrm{PhD}^{1}$, Eric Stephant $\mathrm{MD}^{2}$, Jean-Michel Pontier MD ${ }^{1}$, Pascal Constantin $\mathrm{MD}^{1}$, Christophe Pény $\mathrm{MD}^{1}$.

${ }^{1}$ Département de médecine hyperbare - Hôpital d'instruction des armées Sainte-Anne, 83800 Toulon Armées, France

${ }^{2}$ Département de radiologie - Hôpital d’instruction des armées Sainte-Anne, 83800 Toulon Armées, France

Corresponding author: E. Gempp

gempp@voila.fr

Abstract word count: 252

Main text word count: 2467

Number of references: 23

Number of tables: 3

Number of figures: 2 


\section{ABSTRACT}

Background: Decompression sickness (DCS) affecting the spinal cord is the most dangerous form of diving-related injury with potential sequelae. This study was conducted to evaluate the relationship between spinal cord lesions on MRI and clinical findings in divers with spinal DCS.

Methods: We studied 45 cases of DCS that were referred to our hyperbaric facility with clinical evidence of spinal involvement during the period 2002-2007. The study included only patients who underwent MRI within 10 days of injury. The severity of spinal DCS for each patient was rated numerically for both the acute event and one month later. The presence or absence of back pain was also noted.

Results: Spinal cord lesions were significantly more frequent in divers with severe DCS, and were not seen in any divers who experienced a favorable outcome (Sensitivity $=67 \%$, Specificity $=100 \%$, negative predictive value $=77 \%$, positive predictive value $=100 \%$ ). The presence of vertebral degenerative changes that impinged on the spinal cord was strongly associated with MRI abnormalities but not with a negative outcome. Acute back pain was associated with hyperintense lesions and persistence of neurological sequelae (OR = 14 [95\% CI, 3,1 to 63,5$])$.

Conclusion: The results show that MRI could be helpful in predicting clinical outcome in divers with spinal cord DCS. The presence of medullary compressive factors and vertebral back pain after surfacing indicate increased likelihood of severe myelopathy with incomplete recovery.

Key words: decompression sickness, diving, MRI, disk herniation, spinal cord 


\section{INTRODUCTION}

Divers are at risk of decompression sickness (DCS) resulting from the liberation into blood and tissue of inert gas bubbles previously dissolved within tissues. Injuries to the central nervous system are predominant in DCS and spinal cord is the most commonly affected site (8). The features of spinal involvement are numerous and neurologic symptoms may vary considerably from minimal subjective sensory abnormalities to complete paraplegia with bladder dysfunction. The pathophysiological mechanisms of spinal cord lesions include several hypotheses, i.e. arterial, venous and autochthonous bubble theories, but venous cord infarction resulting from bubble embolization of epidural vertebral venous system causing obstruction of venous drainage and subsequent coagulation activation and platelet aggregation is probably the main explanation for the pathogenesis of this myelopathy $(9,10)$. To date, magnetic resonance imaging (MRI) appears as the most accurate radiologic technique available for the detection of pathologic changes in spinal cord injury (22). However, radiological data on MRI investigation of acute spinal cord DCS are limited $(16,17,21)$ and the prognostic value of this technique needs to be established $(7,13,19)$. Spinal cord damages due to DCS appears generally as hyperintense signals on T2-weighted images, predominantly localized in the upper or middle thoracic cord segment and in the lateral or dorsal white matter columns $(11,13,14,21)$. In few cases, central gray matter is also involved suggesting a more severe pattern of cord damage $(12,15)$. These damages are compatible with experimental and autopsy findings in which observed histopathologic changes consist of punctate hemorrhages, axonal swelling, early demyelination and lacunae possibly due to bubble effects in white matter areas (6).

To our knowledge, a possible link between anatomical compressive factors and the severity of neurologic impairements in spinal cord DCS has never been reported, except for 3 cases 
published by the team research of this paper $(3,4)$. The authors suggested that predisposing factors such as disk herniation or spinal canal narrowing could affect spinal cord off-gassing by altering epidural venous drainage, thus inducing venous stasis and subsequent ischemic myelopathy. Although the neurological manifestations observed initially in spinal cord DCS are frequently accompanied by acute back pain, few studies however emphasized that this symptom was generally followed by aggravation of the disease, and finally could be predictive of poor clinical outcome (1).

Hence, the purposes of this study were to determine, in a larger cohort of DCS patients, whether intramedullary abnormalities detected with early MRI could be correlated with the severity of spinal decompression sickness, and whether these lesions found may be associated with compressive factors of the spinal cord. We also aimed to seek the real prognostic value of the acute back pain preceding neurological manifestations and its relationship with MRI lesions.

\section{PATIENTS AND METHODS}

From June 2002 to October 2007, we performed a retrospective analysis of 65 consecutive divers referred to our hyperbaric center with symptoms indicative of diving-related spinal cord injury. $70 \%$ of the patients were examined with MRI in our department of radiology during the first 1-10 days after the accident (median 3 days). Finally, we reviewed the MR images and the clinical data on 45 divers (38 men and 7 women; mean age (SD) $46 \pm 12$ years; range 22 to 72 years) with spinal cord DCS. The study protocol was approved by the institutional review board.

Clinical diagnosis of spinal cord injury was made when the criteria of bilateral sensory or/and motor deficit was recognized after the diver surfaced. If need be, other characteristic 
symptoms consistent with involvement of spinal cord in DCS such as back pain or bladder dysfunction were recorded. In 8 cases, we also reported a combination of spinal and cerebral or vestibular manifestations.

Diving profiles were as follows: mean maximum depth (SD) $38 \pm 11 \mathrm{msw}$ and mean bottom time (SD) $30 \pm 13$ min. The median time from surfacing to onset of initial symptoms was 2 min (range 0 to $300 \mathrm{~min}$ ) and the median delay to hyperbaric treatment was 3 hours (range 10 $\min$ to $24 \mathrm{~h})$.

Prior to neuroimaging examination, all patients underwent recompression treatment with hyperbaric oxygen and standardized intravenous therapy with administration of methylprednisolone, pentoxyfilline, buflomedil and aspirin according to our hyperbaric center treatment procedures.

MR imaging was performed on 1.5-Tesla magnet (Signa, General Electric Co., Milwaukee, Wisconsin). All examinations of the whole spinal cord included fast-spin-echo T1-weighted sequence in the sagittal plane, and fast-spin-echo T2-weighted in the sagittal and axial planes. Spinal images were obtained with a standard spine coil and parameters were matrix $384 \mathrm{x}$ 128, $\mathrm{TE}=42 \mathrm{~ms}, \mathrm{TR}=2800 \mathrm{~ms}$, four excitations, $3 \mathrm{~mm}$ slice thickness and $0.3 \mathrm{~mm}$ inter-slice interval, for T2-weighted sagittal acquisition; same parameters with $\mathrm{TE}=16 \mathrm{~ms}$, $\mathrm{TR}=650 \mathrm{~ms}$ for T1-weighted images. Field-of-view (FOV) depending on the size of examined area (cervical $24 \times 32 \mathrm{~cm}$, thoracic $36 \times 40 \mathrm{~cm}$ and lumbar $30 \times 32 \mathrm{~cm}$ ).

Initial severity of spinal myelopathy was evaluated according to the gravity score of Boussuges and coworkers, a system which the prognostic value was prospectively validated (5). The score is calculated from five weighted clinical variables as follows: repetitive dive, clinical course before hyperbaric oxygen treatment, objective sensory deficit, motor impairment and urinary disturbance. Significantly cases with scores greater than 7 predicts more severe sequelae than cases with scores of 7 or less. On the basis of the last neurologic 
examination following all hyperbaric treatment, clinical outcome was also determined by the recovery status one month postinjury, i.e. full recovery or presence of residual neurologic symptoms defined as persistent objective sensory, motor or urinary disorders. Disk changes and others abnormal vertebral findings were recorded as pathological if there was a disk or vertebral extension beyond the space between the vertebrae (epidural space).

Data distribution was studied using a Kolmogorov-Smirnov test. All parametric data are presented as mean \pm SD and non-parametric data as median and range. Association of spinal cord lesions with negative outcome and initial severity, and relationship between these MRIdocumented abnormalities with compressive factors and acute back pain, were analysed using Fisher exact test for the group of 45 patients. Calculation of OR with $95 \%$ CI for compression and back pain on sequelae were performed by Woolf's method. Statistic processing was run on Sigmastat 3.0 software program (SPSS inc., Chicago, Illinois). P values less than 0,05 were considered significant.

\section{RESULTS}

Fourteen patients (31 \%) displayed hyperintensities on T2-weighted images in the white matter of spinal cord, in each case corresponding to the neurologic deficit level determined by the initial physical examination. The distribution of lesions was mainly in the posterior part of the column, with an extensive or focal involvement of the cervical and/or thoracic cord segments (fig.1a and 1b).

On the 45 injured divers, 22 (48 \%) presented a severity score exceeded 7 and 21 (46\%) had residual deficits at 1-month. Among the 22 patients with initial high severity score, 19 (86 \%) had incomplete resolution of neurologic symptoms while only 2 divers (8\%) in the group of injured divers with a score of 7 or less presented with residual neurologic features. 
Table I shows that spinal cord lesions detected with MRI were significantly more present in divers with a gravity score greater than $7(\mathrm{p}<0,001)$ and in divers experiencing a poor clinical outcome $(p<0,001)$. The sensitivity and specificity of positive MRI findings to predict sequelae were $67 \%$ and $100 \%$ respectively, with a negative predictive value of $77 \%$ and a positive predictive value of $100 \%$. [table I here]

Fifteen of 45 divers (33 \%) had at least one abnormal disk or vertebral degenerative changes. In 9 cases (20\%), the presence of these vertebral abnormalities on contact with the spinal cord or the existence of syringomyelia (case 2) was strongly associated with spinal cord lesions $(\mathrm{p}<0,006)$ (Table I). All of these cases showed agreement between the level of compression and at least one of the sites of hyperintense images (Table II). Statistical analysis, however, did not display any direct association between existence of anatomical compression and negative outcome. (table III) [table III here] Seventeen divers (38\%) complained of acute back pain immediatly after surfacing or during decompression stops. In 11 of them, the MRI scans showed significantly intramedullary lesions concordant with the level of recorded pain $(\mathrm{p}<0,001)$. Incomplete recovery was achieved in 14 (82 \%) of the 17 patients with this symptom (OR = 14 [95\% CI, 3,1 to 63,5], p $<0,001$ ). (table III)

[table II here]

DISCUSSION

To the best of our knowledge, this is the first report clearly showing a strong relationship between spinal cord lesions revealed by MRI and the severity of neurological DCS in divers. To date, MRI yields low sensitivity in spinal cord DCS, essentially for technical insufficiency to detect small detailed patchy pathologic changes. Compared with previous works, the incidence of intramedullary hyperintense signals in our study seems higher (31 \% vs. 25 \% 
(21) or 8,3 \% (16)), but mean age of divers, image resolution and time interval between diving accident and imaging study were different. Radiological studies evaluating a possible correlation between follow-up MRI and severity of neurologic sequelae are limited, comprising small series and cases reports $(11,19,20,23)$. Moreover, the link between MRI abnormalities and clinical status is not obvious: a normal MRI of spinal cord does not disqualify the diagnosis of DCS $(7,20)$ and improvement in MRI lesions is not systematically associated with improved neurological picture (23). However, MRI findings of spinal cord injury as a result of trauma have been well documented in previous reports and the prognosis of neurological outcome can be predicted based upon the MRI appearance of the spinal cord. An overall generalization of these results is that patients with hemorrhagic cord injuries have the worst neurological outcome, patients with only spinal cord edema do better, and patients with normal appearing spinal cords uniformly have a good outcome (22).

Considering the initial clinical course and the longer-term outcome, MRI of the spinal cord in the present study appears as a very specific method to depict initial damages related with incomplete recovery. However, the lack of signal abnormalities on MRI does not necessarily mean that patient will not develop sequelae because the negative predictive value of a normal investigation is $77 \%$.

In this study, the affected sites of spinal cord are consistent with the MRI findings usually described with predominant involvement of thoracic spinal cord segment. This region is believed to easily lodge nitrogen bubbles due to the low mobility of the thoracic vertebral column (23) and the paucity of blood supply (1). Furthermore, the high-fat content of the myelin with the high solubility of nitrogen in fat explains the typical distribution of the lesions in white matter areas. The significance of these abnormal signals on T2-weighted images remains unclear but some authors have proposed that the nature and extent of spinal lesions could be consistent with a combination of ischemia, cord infarction and perifocal 
vasogenic edema related with the obstruction of epidural venous system $(14,23)$. This notion is also supported by the pathological patterns of the epidural venous plexus in spinal venography after experimental DCS in dogs (9) and spinal cord DCS in man (18) where impairement of venous drainage and congestion are well visualized. An additional finding is that our current data put forward the unexpected role of medullary compressive factors as predictors of negative clinical outcome in subjects presenting spinal cord DCS with positive MRI findings. However, as shown in table III, half of severe spinal cord DCS did not exhibit narrowing of the epidural space suggesting that the sole presence of spinal anatomical compression is not sufficient to worsen DCS course. We hypothetize that these mechanical factors, commonly described as intervertebral disks abnormalities, may impede the venous outflow in the epidural venous plexus, with subsequent accumulation of intravascular bubbles and activation of clotting system, leading to retrograde ischemia of neural tissue. This may result in indirect injury to spinal cord with typical imaging features of venous infarction characterized by a diffuse edema of white matter, corresponding partially to the level of compression. The relationship between the epidural drainage compression in the anterior cord and the finding that most lesions are in the posterior portion of the cord and typically in the white matter appears related to the close connection between the anterior and posterior venous system involving an important horizontal venous network.

It is important to note that these predisposing vertebral abnormalities in our cohort of injured divers were all symptomless, limiting the screening of potential factors increasing risk for spinal cord DCS in this population.

If we exclude 3 of 15 subjects with spinal cord compressive factors other than disk herniation or protrusion (sequelae of vertebral fracture, intramedullary tumor or syringomyelia), the prevalence of abnormal intervertebral disks was lower to the one found in a recent work with 24 divers and 20 control subjects (26 \% vs. $58 \%$ and $38 \%$, respectively) (2) excluding a 
possible selection bias. As suggested in this previous study, our data also may indicate that diving is not associated with increased risk for accumulating disk degeneration.

Of interest is the predominant occurring of a vertebral acute back pain on reaching the surface with MRI abnormalities and also with the persistence of residual neurologic signs. This finding confirms the general impression depicted by previous observations that this early symptom must be considered as a risk factor for the development of severe spinal cord DCS (1). We suggest that this clinical criterion should be included in the severity scoring system of Boussuges as a very specific variable associated with a worse outcome. The origin of this transient manifestation is uncertain but it can be reasonable to state that this condition corresponds probably to an ischemic pain resulting from spinal cord infarction.

We believe that these results need to be ascertained by a prospective study with large series of patients, and that further studies involving DCS animal model could better define the real impact of spinal cord compressive factors on DCS course. Furthermore, progress in image resolution with the development of high field magnet (3 Tesla) in clinical use should improve the sensitivity of MRI in the detection of intramedullary injuries, but also the understanding of the underlying pathophysiologic mechanisms in spinal cord DCS.

\section{CONCLUSION}

An early MRI examination of spinal cord following neurological DCS may provide a major prognostic information about the clinical outcome and the severity of this myelopathy. This technique appears also to be useful in the detection of possible predisposing compressive factors for severe spinal cord DCS development. It is prudent to recommend that high-risk patients with previous cervico-thoracic back pain or spinal trauma undergo radiological investigations with the aim of identifying vertebral abnormalities before diving. Finally, an 
unusual back pain after surfacing should seek immediate medical attention because this early symptom is strongly indicative of serious spinal cord involvement with potential incomplete recovery.

\section{ACKNOWLEGMENTS}

The authors thank the team of the hyperbaric center of Sainte-Anne military hospital for their technical assistance during the course of this study. 


\section{REFERENCES}

1. Aharon-Peretz J, Adir Y, Gordon CR, Kol S, Gal N et al. Spinal cord decompression sickness in sport diving. Arch Neurol 1993;50:753-56.

2. Bartsch T, Cordes P, Keil R, Reuter M, Hutzelmann A et al. Cervico-thoracic disc protrusions in controlled compressed-air diving: clinical and MRI findings. J Neurol 2001;6:514-16.

3. Blatteau JE, Huel JD, Remy FX, Gempp E, Peny C. Cervical spinal cord decompression sickness with paradoxical course. Role of right-to-left shunts and local compression factors. JEUR 2004; 17:153-57. [french]

4. Blatteau JE, Pontier JM, Huel JD. Neurological decompression sickness and syringomyelia. About 2 observations. Bull Med Sub Hyp 2004;14:73-78. [french]

5. Boussuges A, Thirion X, Blanc P, Molenat F, Sainty JM. Neurologic decompression illness: a gravity score. Undersea Hyperb Med 1996;23:151-55.

6. Caruso JL. Pathology of diving accidents. In: Brubbakk AO, Neuman TS (ed). The Bennett and Elliot's Physiology and Medicine of Diving. $5^{\text {th }}$ edn. WB Saunders, London; 2003: 72943.

7. Colineau X, Muyard B, Paoletti H, Dussaut JP, Theobald X et al. Dysbaric neurologic accidents evidenced by MRI. Médecine et armées 1998;26:371-79. [french] 
8. Francis TJR, Mitchell SJ. Pathophysiology of decompression sickness. In: Brubbak AO, Neuman TS (ed). The Bennett and Elliot's physiology and medicine of diving, $5^{\text {th }}$ edn. WB Saunders, London; 2003: 530-56.

9. Hallenbeck JM, Bove AA, Elliott DH. Mechanisms underlying spinal cord damage in decompression sickness. Neurology 1975;25:308-16.

10. Haymaker W, Johnston AD. Pathology of decompression sickness. Mil Med 1955; 117:285-306.

11. Hierholzer J, Tempka A, Stroszczynski C, Amodio F, Hosten $\mathrm{N}$ et al. MRI in decompression illness. Neuroradiology 2000; 42:368-70.

12. Kei PI, Choong CT, Young T, Lee SH, Lim CCT. Decompression sickness: MRI of the spinal cord. J Neuroimaging 2007;17:1-3.

13. Kim MK, Ko SM, Bae JS, Park SS. Spinal decompression sickness. J Neurol Sci 2005;238 (suppl.1): S473-74.

14. Manabe Y, Sakai K, Kashihara K, Shohmori T. Presumed venous infarction in spinal decompression sickness. Am J Neuroradiol 1998;19:1578-80. 
15. Mc Cormac J, Mirvis SE, Cotta-Cumba S, Shanmuganathan K. Spinal myelopathy resulting from decompression sickness: MR findings in a case and review of literature. Emergency Radiology 2002;9:240-42.

16. Moon RE, Massey EW, Debatin JF, Sallee DS, Heinz ER. Radiographic imaging in neurological decompression illness. Undersea Biomed Res 1992; 19 (suppl.):42.

17. Reuter M, Tetzlaff K, Hutzelmann A, Fritsch G, Steffens JC et al. MRI of the CNS in diving-related decompression illness. Acta Radiol 1997;38:940-44.

18. Royon M, Wolkiewiez J. Bilan scanographique et phlébographique d'un accident médullaire de décompression. Med Sub Hyp 1987;4 :117-20. [french]

19. Sparacia G, Banco A, Sparacia B, Midiri M, Brancatelli G et al. Magnetic resonance findings in scuba diving-related spinal cord decompression sickness. MAGMA 1997;5:11115.

20. Tournebise H, Boucand MH, Landi J, Theobald X. Paraplegia and decompression sickness. Paraplegia 1995; 3:636-39.

21. Warren LP, Djang WT, Moon RE, Camporesi EM, Sallee DS et al. Neuroimaging of scuba diving injuries to the CNS. Am J Roentgenol 1988;151:1003-08.

22. White ML, El Khoury GY. Neurovascular injury of spinal cord. Eur Radiol 2002;42:11726. 
23. Yoshiyama M, Asamoto S, Kobayashi N, Sugiyama H, Doi H et al. Spinal cord decompression sickness associated with scuba diving: correlation of immediate and delayed magnetic resonance imaging findings with severity of neurologic impairment-a report on 3 cases. Surgical neurology 2007; 67:283-87. 


\begin{tabular}{ccccc} 
& & MRI + & MRI - & p values \\
\hline \hline \multirow{2}{*}{ Initial severity } & High & 13 & 9 & $<0,001$ \\
& Low & 1 & 22 & \\
\hline \multirow{2}{*}{ Sequelae } & Yes & 14 & 7 & $<0,001$ \\
& No & 0 & 24 & \\
\hline \multirow{2}{*}{ Compression } & Yes & 9 & 6 & $<0,006$ \\
& No & 5 & 25 & \\
\hline \multirow{2}{*}{ Back Pain } & Yes & 11 & 6 & $<0,001$ \\
& No & 3 & 25 & \\
\hline
\end{tabular}

Table I: Association between initial severity score of Boussuges, existence of sequelae at 1 month, anatomical compression of spinal cord, occuring of back pain after surfacing and MRI findings in spinal cord DCS. MRI + indicates the presence of intramedullary hyperintense lesion on T2-weighted imaging. Fisher exact test as appropriate. 


\begin{tabular}{llll}
\hline Case/age & MRI spinal & Compressive factors \\
& & lesion levels
\end{tabular}

1/38 On ascent, tetraparesis followed by aphasia and vomiting, later - ataxia and bladder dysfunction

2/48 After surfacing, thoracic back pain, 5h later paraparesis and bladder dysfunction

3/60 After surfacing, tetraparesthesia, later - ataxia, paraparesis, sensory loss T10 and bladder dysfunction

4/27 After surfacing, thoracic back pain, paraparesis, 30 min later - deterioration with ataxia, paresthesia and bladder dysfunction

5/34 During decompression stop, thoraco-lumbar back pain, paraparesis and paresthesia of lower limbs

6/49 10-min postdive paraparesthesia, later - ataxia, sensory loss T11 and bladder dysfunction

7/64 After surfacing, thoracic back pain, paraparesis, $5 \mathrm{~h}$ later - tetraparesthesia, ataxia, paresis in right arm and bladder dysfunction

After surfacing, loss of consciousness, nausea,

8/56 vomiting, tetraparesthesia, 10h later paraparesis, sensory loss T10 and bladder dysfunction

During decompression stop, thoracic back pain,

9/30 paraparesthesia, 6h later - deterioration with paraparesis, sensory loss T6 level and bladder dysfunction
C6-T11 Disk herniation C6-C7

T10-T12 Disk protrusion C5-C6 with syringomyela T12

C3-C7 Disks herniation C4-C7

C5-T6 Disk herniation C5-C6

T6-T9 Disk herniation T7-T8

C6-T1 Cervical arthritic degeneration with epidural space encroachment C6

C2-C6 and T2 Disks protusion C3-C6 with epidural space encroachment

C4-T12 Osteophytic spurs T6-T7 pushing against epidural space

Sequelae of vertebral fracture

T5-T7 T6-T7 with epidural space encroachment

Table II: Overview of the 9 divers presented MR abnormalities including spinal cord DCS

lesions and medullary compressive factors. 


\begin{tabular}{cccc} 
& Sequelae & Full recovery & p values \\
\hline \hline Compression & 11 & 4 & 0,8 \\
No compression & 10 & 20 & \\
\hline Back pain & 14 & 3 & $<0,001$ \\
No back pain & 7 & 21 &
\end{tabular}

Table III: Association between clinical outcome, presence of spinal cord compressive factor and occuring of back pain after surfacing. Fisher exact test as appropriate. 
FIGURE

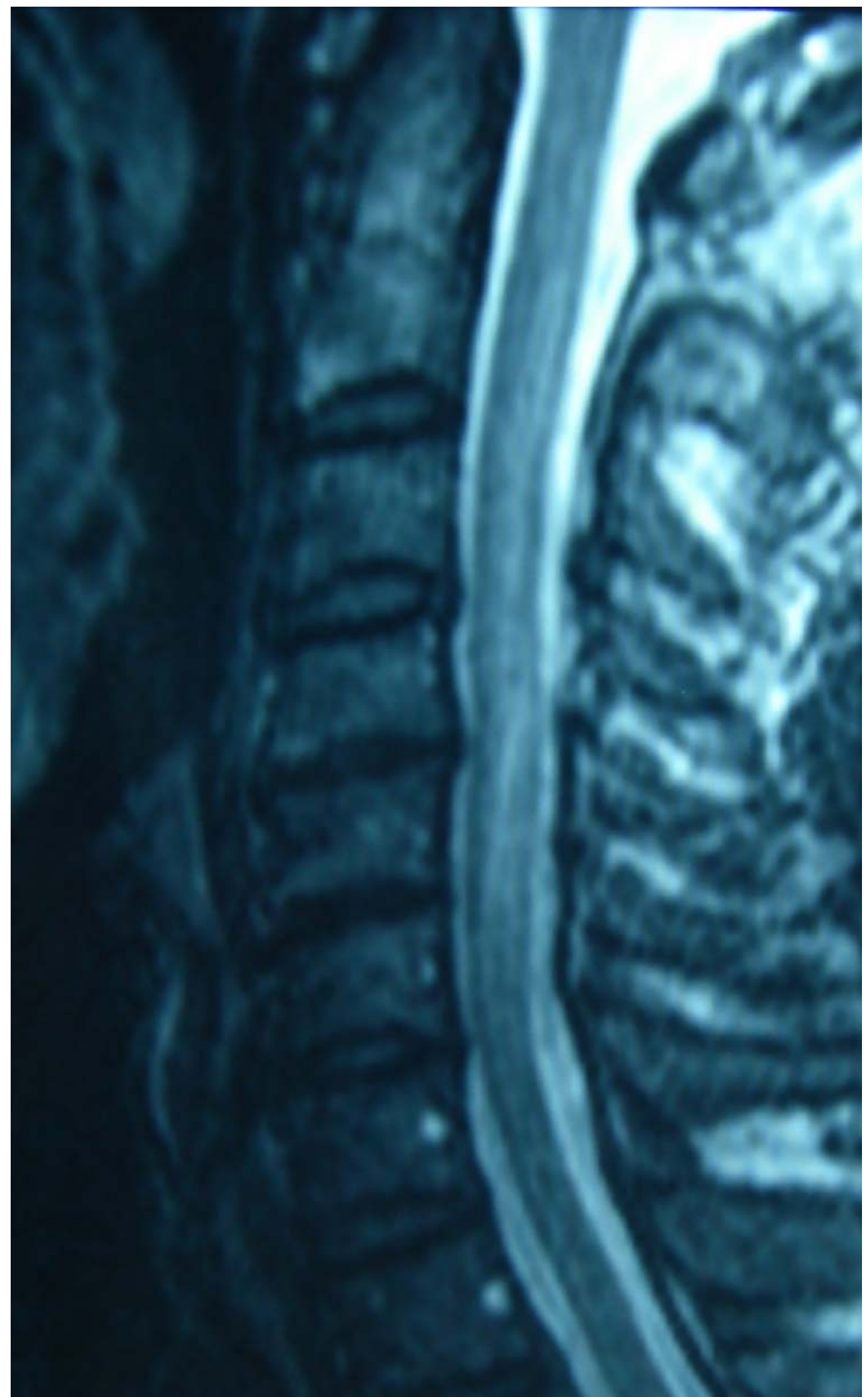

Fig. 1a - Sagittal T2-weighted image of cervical spinal cord (case 7) revealing a hyperintense signal extending from C2 level to the caudal C6 level (white arrows). The picture indicates several disks protrusions with encroachments of epidural space (arrows with dottle line). 


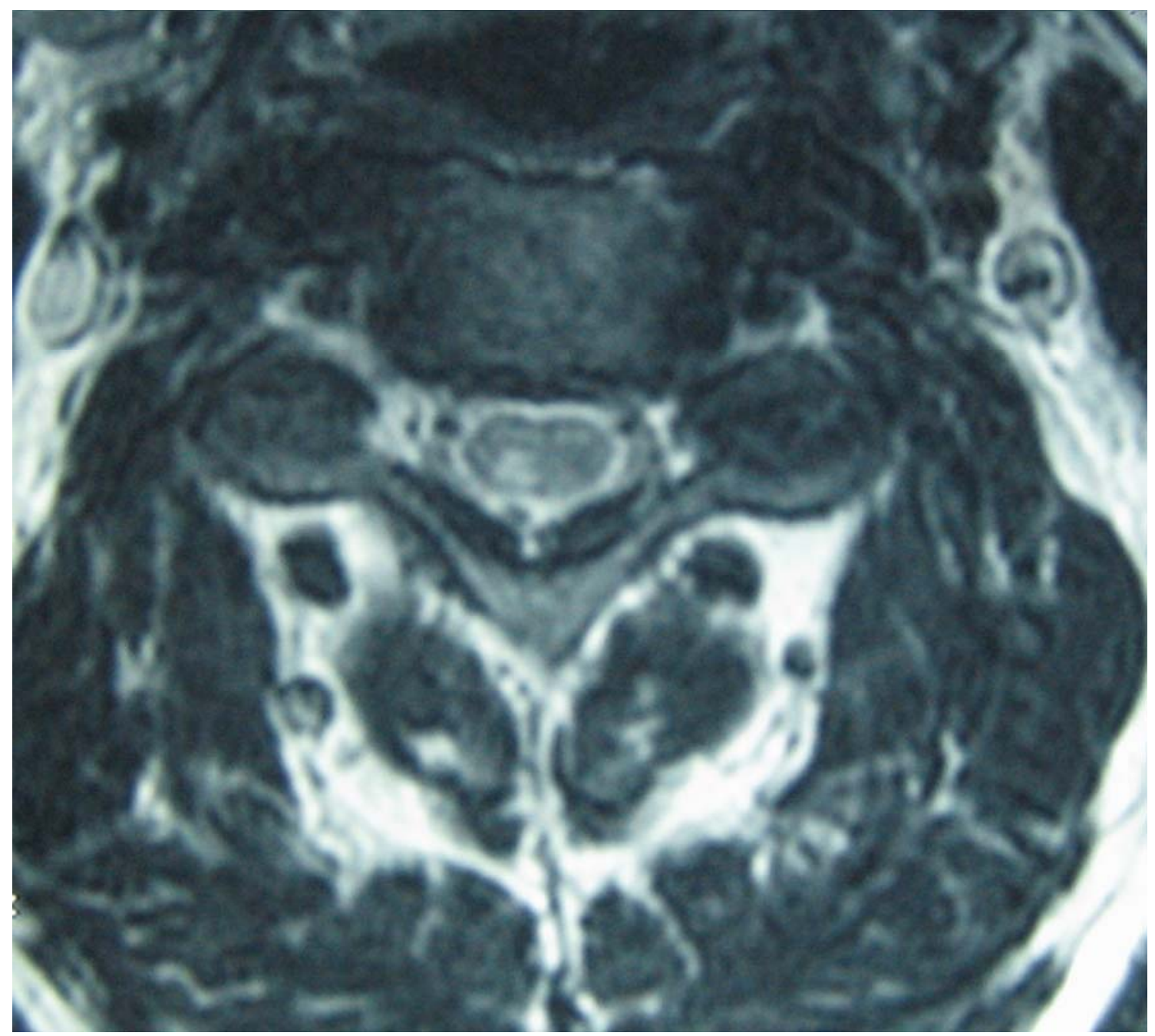

Fig. 1b - Axial T2-weighted image at the C3 level of case 7 showing high-signal abnormality in the dorsal region of the spinal cord (white arrow). 